\title{
Arabic translation and psychometric testing of the self-screening malnutrition universal screening tool in inflammatory bowel disease patients
}

Mahmoud H. Mosli, MD, MSc, Marwan A. Albeshri, MBBS, Anas A. Alsolami, MBBS, Firas A. Addas, MBBS, Abdalrhman M. Qazli, MBBS, Majid Alsahafi, MD, MSc, Hani Jawa, MD, Areej Alkhaldy, PhD, Yousif Qari, MD.

\section{ABSTRACT}

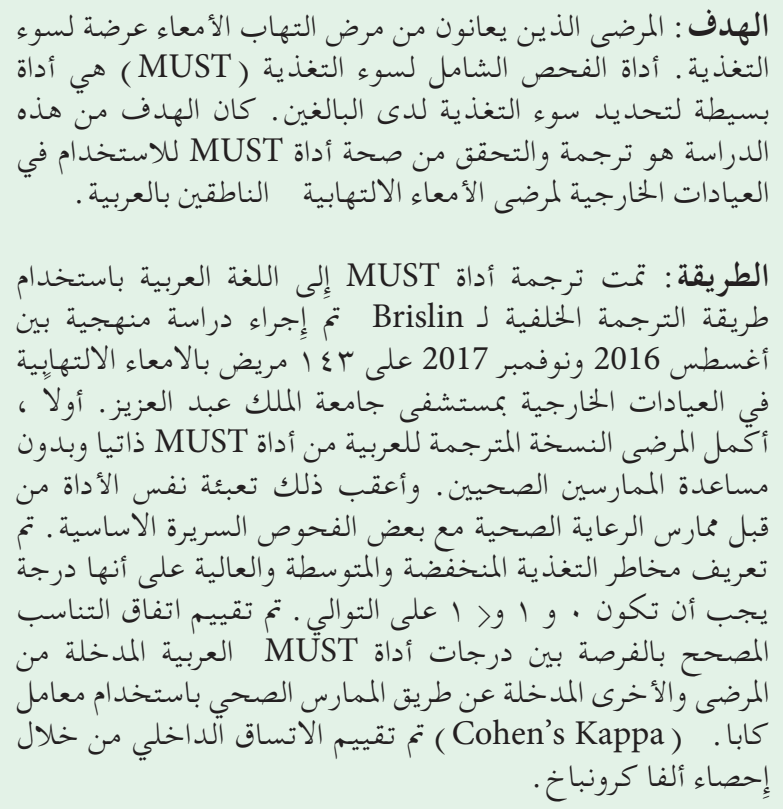

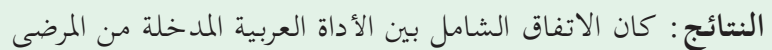

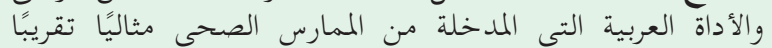

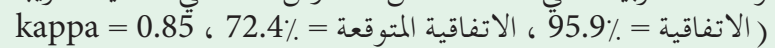

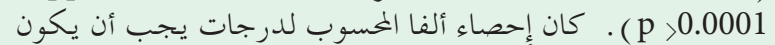

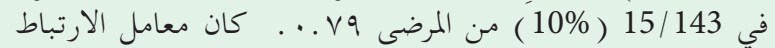

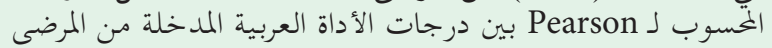

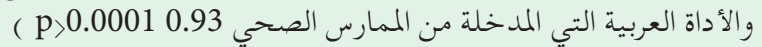

الاستنتاجات : تم العثور على اتفاق مثالي بين أداة MUST الماتلة العربية

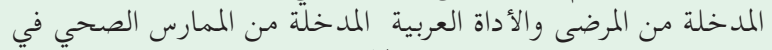

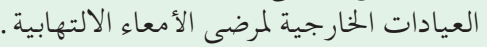

Objectives: To translate and validate the malnutrition universal screening tool (MUST) for use in Arabicspeaking outpatients with inflammatory bowel disease (IBD).
Methods: We translated the MUST into Arabic by using the Brislin back-translation method. This methodological study was performed between August 2016 and November 2017 on 143 outpatients with IBD at King Abdulaziz University Hospital, Jeddah, Saudi Arabia. The patients must completed the selfadministered (PSA) Arabic version of the MUST. Then, the health-care practitioners (HCPs) administered the Arabic MUST and performed standard patient clinical assessment. The nutrition risk was defined as low (MUST score=0), medium (MUST score=1), or high (MUST score $>1$ ). The chance-corrected proportional agreement between the scores from the PSA and HCPadministered Arabic MUST was evaluated using Cohen's kappa coefficient. Internal consistency was evaluated using Cronbach's alpha statistic.

Results: The overall agreement between the PSA and HCP-administered Arabic MUST was almost perfect (agreement $=95.9 \%$, expected agreement $=72.4 \%$, kappa $=0.85, p<0.0001)$. The calculated Cronbach's alpha statistic for MUST scores in 15/143 (10\%) patients was 0.79. The calculated Pearson's correlation coefficient between PSA and HCP-administered MUST scores was $0.93(p<0.0001)$.

Conclusions: The PSA and HCP-administered Arabic MUST showed perfect agreement in outpatients with IBD.

Saudi Med J 2019; Vol. 40 (8): 828-835 doi: 10.15537/smj.2019.8.24427

From the Department of Medicine (Mosli, Alsahafi, Jawa, Qari), from the College of Medicine (Albeshri, Alsolami, Addas, Qazli), and from the Department of Clinical Nutrition (Alkhaldy), King Abdulaziz University, Jeddah, Kingdom of Saudi Arabia.

Received 19th March 2019. Accepted 23rd July 2019.

Address correspondence and reprint request to: Dr. Mahmoud Mosli, Department of Medicine, King Abdulaziz University Hospital, King Abdulaziz University, Jeddah, Kingdom of Saudi Arabia. E-mail:mahmoud.mosli@robartsinc.com 
$\mathrm{I}$ nflammatory bowel disease (IBD) is a chronic immune-mediated disease of unknown etiology that is traditionally considered a disease of the Western world. However, recent studies have shown a trend of increasing IBD incidence in countries previously believed to have a low incidence of the disease, such as the Kingdom of Saudi Arabia (KSA). ${ }^{1,2}$ This increase is believed to be due to a rapid Westernization of lifestyle, including dietary and environmental changes as well as improved sanitation. ${ }^{3}$ Malnutrition is commonly identified in patients with IBD. Malnutrition has been associated with poor clinical outcomes, owing to its negative effects on the physical and psychological well-being of patients with IBD. Moreover, it impairs the recovery from intestinal injury, thereby increasing the morbidity and mortality rates. However, these negative outcomes could be avoided if malnutrition is detected and managed early. ${ }^{4}$ The Subjective Global Assessment (SGA) is a validated tool for assessing malnutrition in different patients, including patients with IBD. It is based on the medical history of patients and physical examination findings, and is considered the gold standard for nutritional assessment. The SGA, however, is highly dependent on physical examination and requires training. The patient-generated SGA, the short form of the SGA, also requires a physical examination component., ${ }^{4,5}$ Unlike the SGA, the Malnutrition Universal Screening Tool (MUST) is a very simple malnutrition screening tool that has been validated in many health-care settings across many patient groups, such as in the oncology, surgical, and medical settings including in outpatients with IBD., The Malnutrition Advisory Group developed MUST as a 5-step tool, and the British Dietetic Association and the British Association for Parenteral and Enteral Nutrition Assessment endorse the use of this tool. ${ }^{6}$ The MUST incorporates body mass index (BMI) scores, degree of weight loss, and effects of acute illness. The final score categorizes a patient as having a low, moderate, or high risk for malnutrition. ${ }^{8}$ Nevertheless, many other validated malnutrition assessment and screening tools are available, although these can considerably add to the workload of health-care workers and may be neglected in busy clinics. Self-assessment tools are considered easy to use and helpful in identifying patients with IBD at a risk for malnutrition in busy clinical settings; however, there are few published works on the ability of patients

Disclosure. Authors have no conflict of interests, and the work was not supported or funded by any drug company. to self-screen for malnutrition. Self-screening has shown good reliability when compared with screening by health-care practitioners (HCPs) for malnutrition and other diseases, and thus could help in treatment., ${ }^{79}$ In 2016, Sandhu et $\mathrm{al}^{10}$ demonstrated high agreement between scores from patient self-administered (PSA) MUST and scores from HCP-administered MUST in patients with IBD. They concluded that the ease of use and high validity of the self-administered MUST would increase the detection of malnutrition in patients with IBD. Currently, there is paucity of validated selfscreening tool in Arabic language for use by outpatients with IBD. Thereby, we aimed to translate the MUST and evaluate its reproducibility, validity, and internal consistency in patients with IBD in the KSA. In this article, we present an Arabic tool capable of evaluating patients with IBD for malnutrition, with clearly outlined operating properties.

Therefore, the objectives of this study were to (i) translate the MUST into Arabic and (ii) to perform psychometric testing through tests of reproducibility, validity, and internal consistency in Arabic-speaking patients with IBD.

Methods. This hospital-based methodological study was conducted at the Gastroenterology Clinics of the Department of Medicine, King Abdulaziz University Hospital (KAUH), Jeddah, Saudi Arabia between August 2016 and November 2017. Outpatients $\geq 18$ years old, diagnosed with IBD, and attending the gastroenterology clinics at KAUH within the study period were considered eligible for inclusion. Pregnant women, mentally or physically impaired patients, and non-Arabic speakers were excluded. The Unit of Biomedical Ethics at King Abdulaziz University Hospital approved the study.

This cross-language validation study involved 2 phases: a translation phase and a validation phase.

Phase 1 - Translation phase. We translated the MUST from English to Arabic by using the Brislin back-translation method, which is a commonly used and widely accepted translation method. ${ }^{11,12}$

The method typically relies on bilingual experts to first translate the questionnaire from the source language to the target language. In this case, the MUST was translated from English to Arabic. Subsequently, another bilingual expert without access to the original version re-translated the tool from Arabic back into English. This back-translated version was then compared to the original English version of the MUST. Repeated translation was completed for any sections that contained errors in meaning or language (Figures $1 \& 2$ ). 
Step 1:
Calculate your body mass index (BMI)
A. Measure your weight (in pounds)
B. Estimate or measure your height (in feet and inches)
C. Use the chart (Appendix 1) to estimate your BMI feet and inches
If your BMI is less than 19 , you score 2 points If your BMI is 19 or 20 , you score 1 point
If your BMI is greater than 20 , you score 0 points

Step 2:

Calculate your weight loss score,

A. What is current weight? pounds

B. Estimate how much weight you have lost in the last 3-6 months?

C. Use the chart (Appendix 2) to estimate your percentage weight loss pounds

If less than $5 \%$ weight loss, your score is 0 points If $5-10 \%$ weight loss, your score is 1 point

If greater than $10 \%$ weight loss, your score is 2 points

Step 3:

A. Do you feel acutely sick right now?

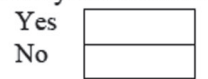

B. Has your intake of food been poor for the last 5 days or likely to be poor for the next 5 days? Yes No

If you answered yes to both questions, then score 2 points. Otherwise, your score is 0 points.

Score:

Step 4:

Add scores together

Overall score:

Step 5:

How easy was this survey? Please circle

Very easy

Easy

Difficult

Very difficult

Figure 1 - English version of the Malnutrition Universal Screening Tool (MUST). BMI - body mass index

Phase 2 - Reliability and internal consistency study. The second phase of the study was conducted at the KAUH gastroenterology outpatient clinics. Patients who met the inclusion criteria and agreed to provide written informed consent were consecutively recruited. The patients were given a package in which they were asked to record demographic information, provide disease-specific information, and complete the PSA MUST as well as an ease-of-use questionnaire. For the ease-of-use questionnaire, the patients were required to select one of 4 responses: very easy, easy, difficult, or very difficult. The patients performed BMI calculations by using a BMI chart. Each room contained an electronic weight measurement scale. The patients estimated or calculated their height in inches and weight in pounds.
They did not receive any further instruction, including any information on the weighing technique. The patients completed the questionnaires in isolation. Once they had completed the MUST screening tool, which included self-calculation of the MUST score, the tool was placed in a sealed envelope. After the completion of the PSA MUST, an HCP (attending physician, medical residents, or medical specialist) assessed the patients and independently administered a MUST assessment while remaining blinded to the PSA scores.

Variables and data collection. By using a standardized and pretested data extraction sheet, we collected the data of all patients from the electronic hospital records. We extracted the following data: age, gender, nationality, IBD subtype, duration of disease in years, 


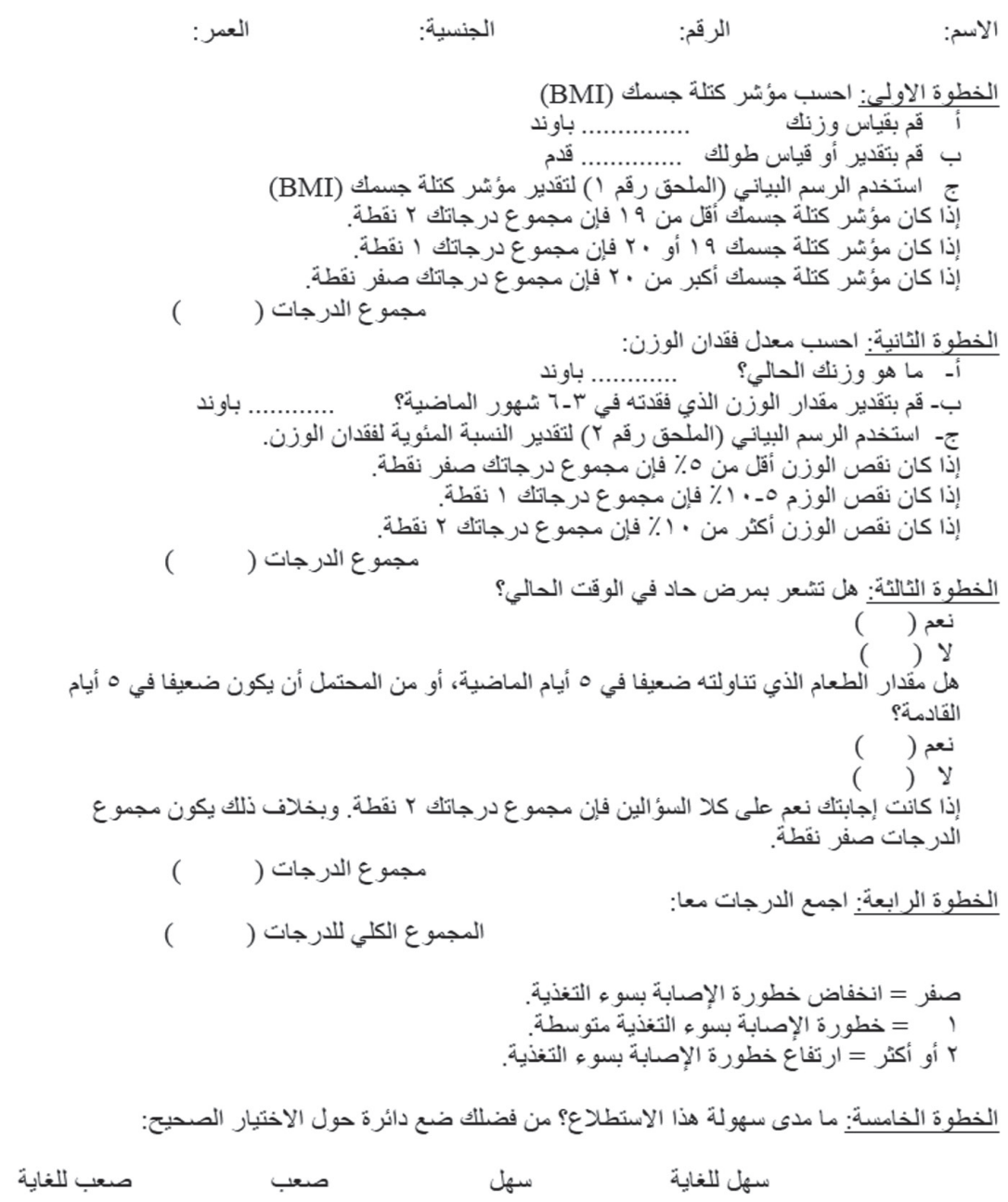

Figure 2 - Arabic version of the Malnutrition Universal Screening Tool (MUST). BMI - body mass index

affected part of the gastrointestinal tract, symptoms, family history of IBD, smoking history, extra-intestinal manifestations, and medications used. We reviewed the endoscopic, histologic, and radiologic reports to confirm the diagnosis of IBD.

Sample size calculation and statistical analysis. Assuming a malnutrition prevalence of $15 \%$ among patients with IBD, a statistical power of $80 \%$, and a 2 -tailed significance level of 0.05 , to test a $\mathrm{K}$ ( 2 categories) of 0.75 against a null hypotheses of $\kappa=$ 0.45 , a sample size of 127 participants was determined. Upon calculating the sample size for reliability, using the non-parametric binomial reliability demonstration test, we found that by testing 120 patients, and allowing 18 possible test failures, we would be able to demonstrate a reliability of $80 \%$ at a $90 \%$ confidence level. In this case, if the item reliability is $\leq 80 \%$, and the chances of passing this test would be $\leq 10 \%$. The sample size for these assumptions would not differ from the sample size we used.

To evaluate chance-corrected agreement between HCP-administered and PSA Arabic MUST scores, we used the benchmarks set by Landis and Koch, which designate weighted kappa values of $<0$ as no agreement, $0-0.20$ as slight agreement, $0.21-0.40$ as fair agreement, $0.41-0.60$ as moderate agreement, 0.61-0.80 as substantial agreement, and $0.81-1$ as almost perfect agreement. ${ }^{13,14}$ We based the agreement on a 2-category classification of MUST scores, as follows: low-risk score (MUST $=0$ ) versus combined medium and high-risk scores (MUST $\geq 1$ ). Additionally, we tested the chance-corrected agreement between 
Table 1 - Baseline characteristics of 143 outpatients with inflammatory bowel disease.

\begin{tabular}{lc}
\hline Baseline characteristic & Summary statistic \\
\hline Mean age, years (SD) & $33(14.7)$ \\
Gender (\%) & $66(46.0)$ \\
Male & $77(54.0)$ \\
Female & \\
Nationality & $112(79.0)$ \\
Saudi & $30(21.0)$ \\
Non-Saudi & \\
Body mass index (mean \pm SD) & $24.6 \pm 6.6$ \\
Mean HCP-administered BMI & $24.9 \pm 6.5$ \\
Mean patient self-administered BMI & \\
MUST score & $1.4 \pm 1.5$ \\
Mean HCP-administered MUST score & $1.4 \pm 1.6$ \\
Mean patient self-administered MUST score & $3(2.0)$ \\
Patient ease-of-use score for patient self-administered MUST score (\%) \\
Very easy & $65(45.0)$ \\
Easy & $72(50.0)$ \\
Difficult & $3(2.0)$ \\
Very difficult & \\
Inflammatory bowel disease type (\%) & $83(58.0)$ \\
Crohn's disease & $57(40.0)$ \\
Ulcerative colitis & $10.7)$ \\
Celiac disease & \\
\hline BHCP - health-care practitioner, MUST - Malnutrition Universal \\
Screening Tool
\end{tabular}

Table 2 - Frequencies of malnutrition categories based on patient- and HCP-administered MUST score

\begin{tabular}{lccccc}
\hline $\begin{array}{l}\text { HCP-administered } \\
\text { MUST scores }\end{array}$ & $\begin{array}{c}\text { Patient self-administered MUST } \\
\text { scores } \\
\text { Low } \\
\text { Intermediate } \\
(\mathbf{0 . 0})\end{array}$ & $\begin{array}{c}\text { High } \\
(\mathbf{1 . 0})\end{array}$ & ( 1) \\
\hline Low $(0.0)$ & 51 & 0 & & 2 & $(53)$ \\
Intermediate (1.0) & 2 & 28 & & 2 & $(32)$ \\
High $(>1)$ & 6 & 0 & 52 & $(58)$ \\
Total & 59 & 28 & 56 & 143 & $(100)$ \\
\hline \multicolumn{4}{r}{ HCP - health-care practitioner, PSA - patient self-administered } \\
\hline
\end{tabular}

PSA and HCP-administered Arabic MUST scores for all 3 categories (low-, medium-, and high-risk scores), measured with $95 \%$ confidence intervals (95\% CIs). ${ }^{15}$ Pearson's correlation coefficient (r) was used to calculate the correlation (validity) between the PSA and HCPadministered MUST and BMI scores.

Internal consistency (the extent to which a concept has a consistent measurement) was evaluated using the MUST scores of a study subsample of $35 / 143$ patients (24\%). Cronbach's alpha statistic, which ranges from 0 to 1 , was used to measure internal consistency in which a higher score reflects higher covariance between scale items and a higher probability that they measure the same statistic.
Table 3 - Agreement between patient self-administered and health-care practitioner-administered MUST and BMI scores based on kappa statistic.

\begin{tabular}{lcccc}
\hline Scores & Agreement & $\begin{array}{c}\text { Expected } \\
\text { agreement }\end{array}$ & Kappa & $P$-value \\
\hline MUST score & $96.0 \%$ & $72.4 \%$ & 0.85 & $<0.0001$ \\
BMI score & $19.9 \%$ & $47.0 \%$ & 0.18 & $<0.0001$ \\
\hline \multicolumn{5}{c}{ MUST - malnutrition universal screening tool, } \\
BMI - body mass index \\
\hline
\end{tabular}

Table 4 - Correlation between patient- and HCP-generated scores based on Pearson's correlation coefficient

\begin{tabular}{lcc}
\hline Administered score & $\begin{array}{c}\text { HCP- } \\
\text { administered } \\
\text { MUST }\end{array}$ & $\begin{array}{c}\text { HCP- } \\
\text { administered } \\
\text { BMI }\end{array}$ \\
\hline Patient self-administered MUST & $\begin{array}{c}0.93 \\
(p<0.0001)\end{array}$ & - \\
Patient self-administered BMI & - & 0.94 \\
& \multicolumn{2}{c}{$(p<0.0001)$} \\
\hline HCP - health-care practitioner, MUST - Malnutrition Universal \\
Screening Tool, BMI - body mass index
\end{tabular}

We performed reliability testing and test-retest analysis on a study subsample of $15 / 143$ patients (10\%), which provided separate measurements of BMI and MUST scores at 2 time points at least 2 weeks apart.

We used univariable linear regression analysis to test associations between MUST score and all independent patient variables. Multiple regression analysis was used only when appropriate. Accordingly, regression coefficients (coeff) with 95\% CIs were generated. STATA 12.1 (Stata Corp, College Station, TX, USA) was used for statistical analysis, and $p<0.05$ was considered statistically significant.

Results. Baseline characteristics. A total of 143 outpatients with IBD were recruited for this study. Of them, 83 patients (58\%) had Crohn's disease, 57 patients $(40 \%)$ had ulcerative colitis, and one patient $(0.7 \%)$ had celiac disease. The mean age $( \pm$ SD) for our patients was $32.7( \pm 14.7)$ years. Sixty-six patients were men (46\%) and 77 (54\%) were women (Table 1).

On the basis of the MUST scores recorded by HCPs, $37 \%$ of the patients $(\mathrm{n}=53)$ were classified as having a low risk (MUST score $=0), 22 \%(n=32)$ a medium risk (MUST score $=1)$, and 41\% ( $\mathrm{n}=58)$ a high risk (MUST score $\geq 2$ ) for malnutrition. In comparison, the Arabic PSA MUST screening classified $41 \%$ of the patients $(\mathrm{n}=59)$ as having a low risk, $20 \%(\mathrm{n}=28)$ a medium risk, and $39 \%(n=56)$ a high risk for malnutrition (Table 2). 
Table 5 - Item test-retest (inter-item) correlations between patient- and HCP-generated MUST scores for 15 paired measurements.

\begin{tabular}{|c|c|c|c|c|}
\hline Item & $\begin{array}{c}\text { HCP-administered } \\
\text { MUST } 1\end{array}$ & $\begin{array}{c}\text { Patient-administered } \\
\text { MUST } 1\end{array}$ & $\begin{array}{c}\text { HCP-administered } \\
\text { MUST } 2\end{array}$ & $\begin{array}{c}\text { Patient-administered- } \\
\text { MUST } 2\end{array}$ \\
\hline HCP-administered MUST $1^{*}$ & 1.00 & - & - & - \\
\hline Patient self-administered MUST $1^{*}$ & 0.99 & 1.00 & - & - \\
\hline HCP-administered MUST $2^{*}$ & 0.19 & 0.25 & 1.00 & - \\
\hline Patient-administered MUST 2* & 0.19 & 0.25 & 1.00 & 1.00 \\
\hline
\end{tabular}

According to the preset criteria, the overall agreement between the PSA and HCP-administered Arabic MUST scores (inter-rater agreement) was almost perfect (agreement $=95.9 \%$, expected agreement $=72.4 \%$, kappa $=0.85, p<0.0001)$; however, the agreement between the PSA MUST score and HCPadministered Arabic BMI scores (inter-rater agreement) was poor (agreement $=19.9 \%$, expected agreement $=$ $47 \%$, kappa $=0.18, p<0.0001$; Table 3).

In the test-retest analysis, the inter-class correlation coefficient between the $2 \mathrm{HCP}$-administered Arabic MUST scores was $0.30 \quad(95 \%$ CI $-1.272-0.773$, $p=0.265)$ and that between the 2 PSA MUST scores was 0.394 (95\% CI $-968-802, p=0.193)$.

The calculated Cronbach's alpha statistic for MUST scores was 0.79 (Table 2) and that for BMI scores was 0.961 (Table 4).

The calculated Pearson's correlation coefficient between the PSA and HCP-administered MUST scores was $0.93(p<0.0001)$, and that between the PSA and HCP-administered BMI scores was $0.94 \quad(p<0.0001$; Table 5).

In linear regression analysis, the HCP-administered MUST score was predicted by the HCP-administered BMI (coeff $=-0.06, p=0.031$ ), clinical picture (coeff $=0.44, p=0.008)$, smoking, $($ coeff $=-0.05, p=0.04)$, and albumin level $($ coeff $=-0.05, p=0.05)$.

Discussion. Patients with IBD have an increased risk for malnutrition, which could influence the disease management and outcomes. ${ }^{4}$ The MUST is widely used in general practice in many parts of the world, especially in Europe, and is becoming a more popular malnutrition screening tool owing to its relatively simple application. ${ }^{8}$

Previous studies have demonstrated that selfscreening of outpatients with IBD for malnutrition risk by using the simple 5-step MUST was both reliable and valid.10 In this study, we demonstrated the validity of the Arabic-translated MUST for patients with IBD. Moreover, we showed that an Arabic-translated version of the MUST is reproducible (kappa $=0.85, p<0.0001$ ) and valid (Pearson's correlation coefficient $=0.93$, $p<0.0001)$. According to the Cronbach's alpha statistic (0.79 for MUST scores), the Arabic version also has high internal consistency. In comparison, the reproducibility of BMI, which is seldom used to make quick judgments about nutritional status, appeared to be poor (kappa = $0.18, p<0.0001)$ when the measurement was conducted in Arabic. However, most of our patients were not familiar with calculating their BMI and found it difficult to interpret the figures provided to calculate their BMI. We hypothesize that this could be the reason behind the observed discrepancies in BMI measurements collected by HCP versus patients. However, BMI is only one item in the MUST score and so the difference should not affect the total MUST score of each patient. This may be reflective of the inability of patients to conduct proper measurements of their own height and weight. Krul et a ${ }^{16}$ demonstrated differences in self-reported and measured height and weight between women and men. Women mostly under-reported their weight and overreported their height, causing BMI underestimation. On the other hand, men mostly overestimated their height, also causing BMI underestimation.

Recent studies have demonstrated that HCPdocumented MUST scores are an accurate and clinically meaningful reflection of nutritional status. MUST scoring has been used to evaluate adult and pediatric patients with a variety of gastrointestinal disorders, including IBD. Furthermore, patient-driven MUST assessments in outpatients with IBD have been shown to correlate well with those administered by HCPs, showing reliable and valid results. ${ }^{10}$

With IBD emerging as an increasing problem worldwide, it is essential to develop tools for the assessment of non-English-speaking patients that are operated using their native language, as non-valid methods of translation can lead to misinterpretation of results and ultimately clinical errors. Over the past 2 decades, IBD has appeared in the Arabic peninsula and in Northern Africa, 2 geographical areas predominantly inhabited by Arabic-speaking people. ${ }^{17,18}$ As only small percentages of people living in these areas speak and 
understand English, it is essential to develop accurate Arabic assessment tools that can be used in daily practice. Proof of concept had already been demonstrated for this approach in a study that used the dialysis symptom index in patients with chronic kidney disease, which reported excellent validity of the Arabic-translated tool. ${ }^{19}$ The results from our current study in patients with IBD provide further evidence for the validity of this approach.

It is important to be able to identify clinical predictors of malnutrition with simple clinical parameters. Nguyen et $\mathrm{al}^{20}$ examined hospitalized patients with IBD and identified fistulizing Crohn's disease (odds ratio [OR] 1.65, 95\% CI 1.50-1.82) and a history of bowel resection (OR 1.37, 95\% CI 1.27-1.48) as significant predictors of malnutrition. 20 Furthermore, Csontos et al. performed a cross-sectional survey of 173 outpatients with IBD who were screened using the MUST, and reported that $21.4 \%$ of the patients were at risk of malnutrition and $27.7 \%$ were at risk of sarcopenia. ${ }^{21}$ In a prospective multicenter study from Spain that evaluated 1271 outpatients with IBD, Casanova et $\mathrm{al}^{22}$ reported a malnutrition prevalence of 16\% (95\% CI 12-20\%). According to their multivariate analysis, a history of abdominal surgery, active disease, and dietary restrictions during flares were associated with a higher risk of malnutrition. ${ }^{22}$ We sought to identify the predictors of malnutrition according to MUST scores, and our regression analysis identified BMI (coeff $=-0.06, p=0.031$ ), clinical picture (coeff $=0.44, p=0.008)$, smoking $($ coeff $=-0.05, p=0.04)$, and albumin level (coeff $=-0.05, p=0.05$ ) as relevant predictors of malnutrition. ${ }^{21}$

The results of this study strongly support the conclusion that the Arabic version of the MUST is reliable and valid; however, the study is limited by the small sample size of outpatients with IBD, drawn from a single study center. Future prospective studies aimed at evaluating the use of the Arabic-translated MUST questionnaire in outpatients with IBD are needed to externally validate our results.

We conclude that this Arabic version of the MUST carries operational properties that are similar to the English version, reflecting its validity. The agreement between the PSA MUST completed in Arabic and the HCP-administered MUST is almost perfect. The self-administered MUST is an easy-to-use and valid tool that, following further validation in Arabic, could increase the early detection of malnutrition risk in outpatients with IBD.
Acknowledgment. We would like to thank Editage (www. editage.com) for English language editing.

\section{References}

1. El Mouzan MI, Saadah O, Al-Saleem K, Al Edreesi M, Hasosah $\mathrm{M}$, Alanazi A, et al. Incidence of pediatric inflammatory bowel disease in Saudi Arabia: a multicenter national study. Inflamm Bowel Dis 2014; 20: 1085-1090.

2. Al-Bawardy B. Inflammatory bowel disease: clinical screening and transition of care. Saudi J Gastroenterol 2017; 23: 213.

3. Ye Y, Pang Z, Chen W, Ju S, Zhou C. The epidemiology and risk factors of inflammatory bowel disease. Int J Clin Exp Med 2015; 8: 22529.

4. Valentini L, Schulzke JD. Mundane, yet challenging: the assessment of malnutrition in inflammatory bowel disease. Eur J Intern Med 2011; 22: 13-15.

5. Mijac DD, Janković GL, Jorga J, Krstić MN. Nutritional status in patients with active inflammatory bowel disease: prevalence of malnutrition and methods for routine nutritional assessment. Eur J Intern Med 2010; 21: 315-319.

6. Elia M. Screening for malnutrition: a multidisciplinary responsibility. Development and use of the Malnutrition Universal Screening Tool ('MUST') for adults. Redditch: BAPEN; 2003.

7. Cawood AL, Walters ER, Sharp SKE, Elia M, Stratton RJ. 'Selfscreening' for malnutrition with an electronic version of the Malnutrition Universal Screening Tool ('MUST') in hospital outpatients: concurrent validity, preference and ease of use. $\mathrm{Br} J$ Nutr 2018; 120: 528-536.

8. Stratton RJ, Hackston A, Longmore D, Dixon R, Price S, Stroud M, et al. Malnutrition in hospital outpatients and inpatients: prevalence, concurrent validity and ease of use of the 'malnutrition universal screening tool' ('MUST') for adults. $\mathrm{Br}$ J Nutr 2004; 92: 799-808.

9. Keetarut K, Zacharopoulou-Otapasidou S, Bloom S, Majumdar A, Patel PS. An evaluation of the feasibility and validity of a patient-administered malnutrition universal screening tool ('MUST') compared to healthcare professional screening in an inflammatory bowel disease (IBD) outpatient clinic. J Hum Nutr Diet 2017; 30: 737-745.

10. Sandhu A, Mosli M, Yan B, Wu T, Gregor J, Chande N, et al. Self-screening for malnutrition risk in outpatient inflammatory bowel disease patients using the Malnutrition Universal Screening Tool (MUST). JPEN J Parenter Enteral Nutr 2016; 40: 507-510.

11. Brislin RW. Back-translation for cross-cultural research. $J$ Cross-cult Psychol 1970; 1: 185-216.

12. Cha ES, Kim KH, Erlen JA. Translation of scales in crosscultural research: issues and techniques. J Adv Nurs 2007; 58 : 386-395.

13. Landis J, Koch G. The measurement of observer agreement for categorical data. Biometrics 1977; 33: 159-174.

14. Cawood A, Elia M, Sharp S, Stratton R. Malnutrition selfscreening by using MUST in hospital outpatients: validity, reliability, and ease of use. Am J Clin Nutr 2012; 96: 10001007.

15. Donner A. Approaches to sample size estimation in the design of clinical trials-a review. Stat Med 1984; 3: 199-214.

16. Krul AJ, Daanen HA, Choi H. Self-reported and measured weight, height and body mass index (BMI) in Italy, the Netherlands and North America. Eur J Public Health 2011; 21: 414-419. 
17. Farrukh A, Mayberry JF. Epidemiology of inflammatory bowel disease in the Middle East-an opportune time. Arab J Gastroenterol 2014; 15: 163-165.

18. Ng SC, Shi HY, Hamidi N, Underwood FE, Tang W, Benchimol EI, et al. Worldwide incidence and prevalence of inflammatory bowel disease in the 21st century: a systematic review of population-based studies. Lancet 2018; 390: 2769-2778.

19. Almutary H, Bonner A, Douglas C. Arabic translation, adaptation and modification of the dialysis symptom index for chronic kidney disease stages four and five. BMC Nephrol 2015; 16: 36 .
20. Nguyen GC, Munsell M, Harris ML. Nationwide prevalence and prognostic significance of clinically diagnosable proteincalorie malnutrition in hospitalized inflammatory bowel disease patients. Inflamm Bowel Dis 2008; 14: 1105-1111.

21. Csontos Á, Molnár A, Piri Z, Pálfi E, Miheller P. Malnutrition risk questionnaire combined with body composition measurement in malnutrition screening in inflammatory bowel disease. Rev Esp Enferm Dig 2017; 109: 26-32.

22. Casanova MJ, Chaparro M, Molina B, Merino O, Batanero R, Dueñas-Sadornil C, et al. Prevalence of malnutrition and nutritional characteristics of patients with inflammatory bowel disease. J Crohns Colitis 2017; 11: 1430-1439.

\section{Student Corner}

We invite students from a variety of medical disciplines to submit original contributions based on their supervised research.

The Student Corner of Saudi Med J aims to help students explore research opportunities and network with other peers and mentors in the same field.

Submission Guidelines

Submitted Abstracts should include the following:

- Title should be descriptive

- Author's names and affiliation(specify college level/year, academic degree of Senior Author)

- Abstract must be structured and not more than 300 words

- The following are the typical headings:

Objectives (background, why the study was done, specific aims)

Methods (setting, date of study, design, subjects, intervention and analysis)

Results (findings, data and statistical tests) and

Conclusion (general interpretation of results)

General Information on Abstract Submission

Submitted Abstracts should be co-authored by a Senior Supervisor

Abstracts will be reviewed by Student's Corner Section Editor

There is no fee to submit an Abstract

Ethical Approval should be provided

Non-indexed materials 